\title{
Multi-year statistical and modelling analysis of submicrometer aerosol number size distributions at a rain forest site in Amazonia
}

Luciana Varanda Rizzo ${ }^{1}$, Pontus Roldin ${ }^{2}$, Joel Brito ${ }^{3, *}$, John Backman ${ }^{4, * *}$, Erik Swietlicki², Radovan Krejci ${ }^{5}$, Peter Tunved ${ }^{5}$, Tukka Petäjä ${ }^{4}$, Markku Kulmala ${ }^{4}$, and Paulo Artaxo ${ }^{3}$

${ }^{1}$ Departamento de Ciências Ambientais, Universidade Federal de São Paulo, Diadema, Brazil

${ }^{2}$ Physics Institute, Lund University, Lund, Sweden

${ }^{3}$ Instituto de Física, Universidade de São Paulo, São Paulo, Brazil

${ }^{4}$ Department of Physics, University of Helsinki, Helsinki, Finland

${ }^{5}$ Department of Environmental Science and Analytical Chemistry (ACES), Stockholm University, Stockholm, Sweden

* now at: Laboratory for Meteorological Physics, Université Clermont Auvergne, Clermont-Ferrand, France

** now at: Finnish Meteorological Institute, Atmospheric Composition Research, Helsinki, Finland

Correspondence to: Luciana V. Rizzo (lrizzo@unifesp.br) 


\section{S1 Characteristics of model estimated particle sources that reproduce the observed diurnal behavior of particle number size distributions}

Figure S6 shows the estimated daily median contribution to the particle number size distribution in the lowermost 1000 meters of the atmosphere from the Type 1 source of particles emitted at the surface, the Type 2 source of particles entrained into the

5 CBL during the morning, and the Type 3 source of particles from convective downdrafts, during the wet (a) and dry season (b). During the wet season, both the fitted Type 1 and Type 2 particle sources contains a clear nucleation mode, and to a less extent also in Type 3. The estimated median particle number size distribution in convective downdrafts (Type 3) is similar to the observed particle number size distributions by Wang et al. (2016) for convective downdraft events.

Figure S7 shows the diurnal variations in the modelled particle source strength of Types 1 to 3 . The Type 1 surface source strength is largest during the afternoon and evening 16:00-20:00 LT. Because of the shallow NL, this particle source have large impact on the particle number size distribution during the night and early morning. The entrainment of accumulation mode particles (Type 2) contributes to the particle number concentration in the BL during the morning hours, when the CBL height is increasing steeply. In the afternoon (12:00-16:00 LT), especially during the wet season, the dominating particle source in the BL is caused by convective downdrafts containing mainly Aitken mode particles. This explains the decreasing observed and modelled particle geometric mean diameter during the afternoon.

Figure S8 shows how the modelled particle number size distribution changes if we consider a hypothetical convective precipitation event between 14:00 and 14:15 with a rainfall intensity of $20 \mathrm{~mm} \mathrm{~h}^{-1}$. The model results are qualitatively in agreement with the observed particle number size distribution evolution during the convective rainfall events analysed by Wang et al. (2016) and with the average observations from the 86 precipitation events presented in Figure 8 . The modelled total particle number concentration decreases because of the convective downdrafts, which brings to the BL cleaner air mass, and to a less extent, because of the below cloud scavenging. The model also predicts a net decrease of particles larger than $100 \mathrm{~nm}$ in diameter and a small increase of particles below $50 \mathrm{~nm}$ in diameter, in agreement with the observations presented in Figure 8.

\section{S2 Predominance of particle size distribution cluster \#7 - two case studies}

25 In the dry season, particle size distributions were dominated by the accumulation mode. Although, clusters analysis indicated a distinction between two accumulation mode dominated size distributions: cluster \#5, with a centroid peaking at $140 \mathrm{~nm}$, and cluster \#7, with a centroid peaking at $210 \mathrm{~nm}$ (Fig. 5b). Analysis of support variables indicate that cluster \#7 occurred under relatively high values of particle scattering and absorption coefficients (on average, respectively, $55 \mathrm{Mm}^{-1}$ at $550 \mathrm{~nm}$, and 4.5 $\mathrm{Mm}^{-1}$ at $637 \mathrm{~nm}$ ), and in the driest (average RH of 68\%) (Fig. 6d), warmer (average temperature of $29^{\circ} \mathrm{C}$ ) and windy (average wind velocity of $2.3 \mathrm{~m} \mathrm{~s}^{-1}$ ) days of the time series.

Here, two periods of size distribution cluster \#7 predominance were selected to investigate the factors that may lead to this predominance over the other dry season clusters \#5 and \#6. Biomass burning fire counts and Hysplit back trajectories were used for that. Fire spots were detected by the polar satellites NOAA 15-19 and AQUA, and by the geostationary satellite GOES- 
12, operationally processed by the Brazilian National Institute of Spatial Research (CPTEC/INPE). A fire spot indicates the occurrence of fire in one pixel, i.e., $1-20 \mathrm{~km}^{2}$ depending on the satellite resolution, and does not carry information about the burning intensity or extent. The fire counts presented in this work can be overestimated, since the same burning event may occasionally be detected by two satellites in slightly different positions. Daily and 5-day fire spots were computed for three areas: the whole Amazon Basin; a square NE to the TT34 site (latitude $-3.2^{\circ}$ to $0.0^{\circ}$; longitude $-60.5^{\circ}$ to $-56.0^{\circ}$ ); and a square representing areas neighbor to the TT34 site (latitude $-3.2^{\circ}$ to $-2.2^{\circ}$; longitude $-61.0^{\circ}$ to $-59.0^{\circ}$ ). Hysplit back trajectories were calculated for every hour, starting at $100 \mathrm{~m}$ height and over 48 hours. GDAS (Global Data Assimilation System) wind fields with $1^{\circ}$ resolution were used in the calculations.

Figure S11 refers to the dry season of 2009, when cluster \#7 predominated along several days. Figure S11 a) and d) indicate a profusion of fire spots relatively close to the TT34 site when the cluster \#7 prevailed. Before that, in August of 2009, the cluster \#5 predominated, and the fire spots were scarcer and more distant from the TT34 site. Figure S12 refers to a strong biomass burning event observed in 18-23 August 2014, when fire spots in Amazonia reached 14000 counts day ${ }^{-1}$. Back trajectories from SE, where a profusion of fire spots were detected, indicate the transport of biomass burning aerosols to the TT34 site, resulting in elevated particle number concentrations $\left(4800 \mathrm{~cm}^{-3}\right)$, scattering coefficients $\left(180 \mathrm{Mm}^{-1}\right.$ at $\left.550 \mathrm{~nm}\right)$ and absorption coefficients $\left(7.7 \mathrm{Mm}^{-1}\right.$ at $\left.637 \mathrm{~nm}\right)$. Cluster \#7 predominated during this biomass burning event. After the event, fire spots were detected farther from the TT34 site, and clusters \#5 and \#6 prevailed.

These two case studies suggest that particle size distribution cluster\#7 may be connected to the influence of fresh biomass burning aerosols. On the other hand, the dry season clusters \#5 and \#6 may be associated to the influence of aged biomass burning aerosols. Considering the whole time series, clusters \#5 and \#6 were more frequent than cluster \#7, indicating that regional and relatively aged biomass burning emissions may be the major anthropogenic influence over the TT34 site in the dry season. Clusters \#5 and \#6 also shows some contribution from the Aitken mode, which were prominent in the wet season clusters, suggesting a superposition of biomass burning and biogenic aerosols. 

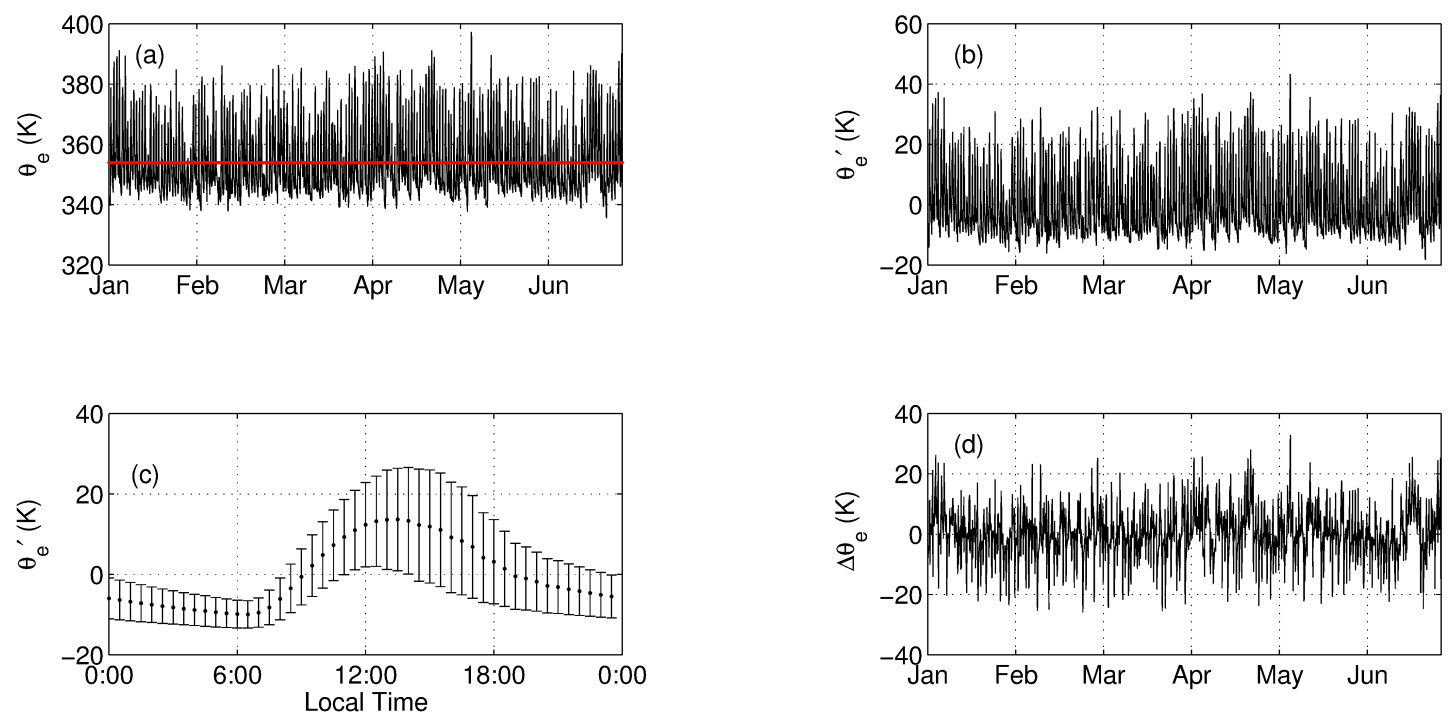

Figure S1. Illustration of the procedure to obtain the time series of $\Delta \theta_{e}$, which is the deviation of observed $\theta_{e}$ from its mean diurnal behavior at a particular season. Wet season of 2009 is used as an example. (a) Time series of $\theta_{e}$ (black) and its seasonal mean (red). (b) Time series of $\theta_{e}$ detrended by its seasonal mean $\left(\theta_{e}^{\prime}\right)$. (c) Mean diurnal cycle of $\theta_{e}^{\prime}$. (d) Time series of $\Delta \theta_{e}$, determined by subtracting $\theta_{e}^{\prime}$ values from their seasonal mean diurnal cycle. 


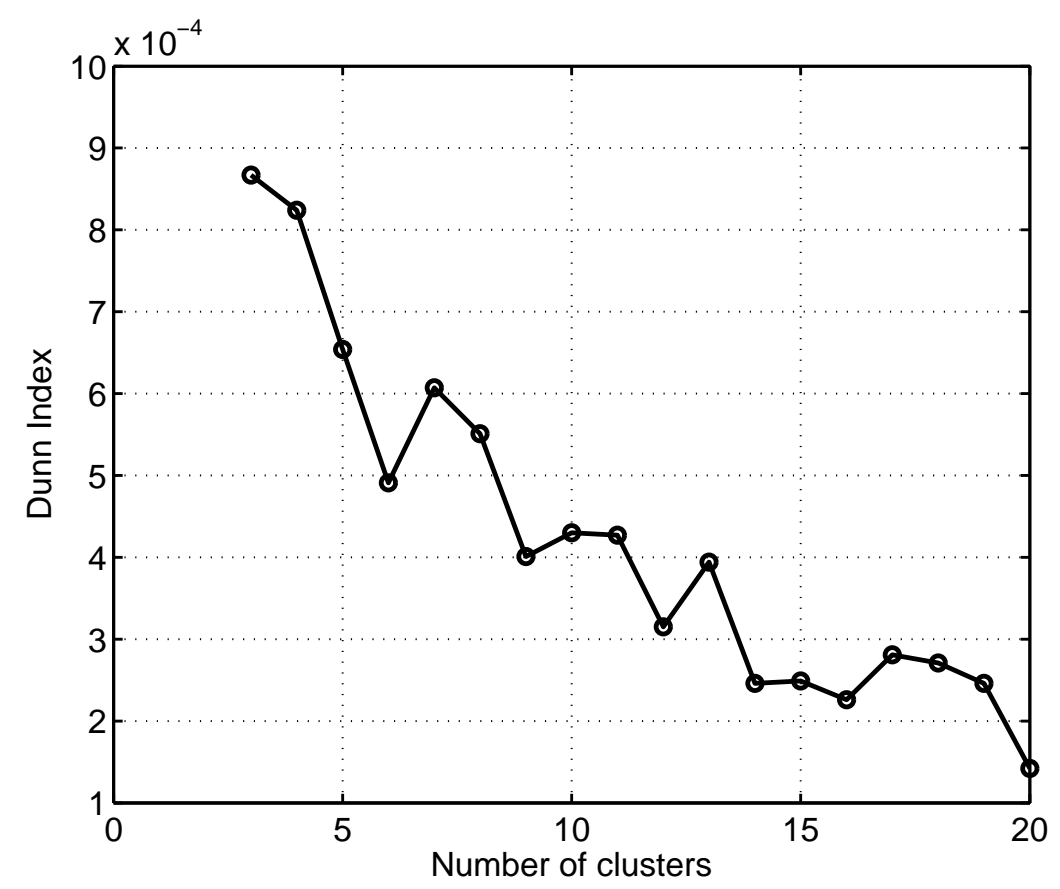

Figure S2. Dunn index as a function of the number of clusters. 

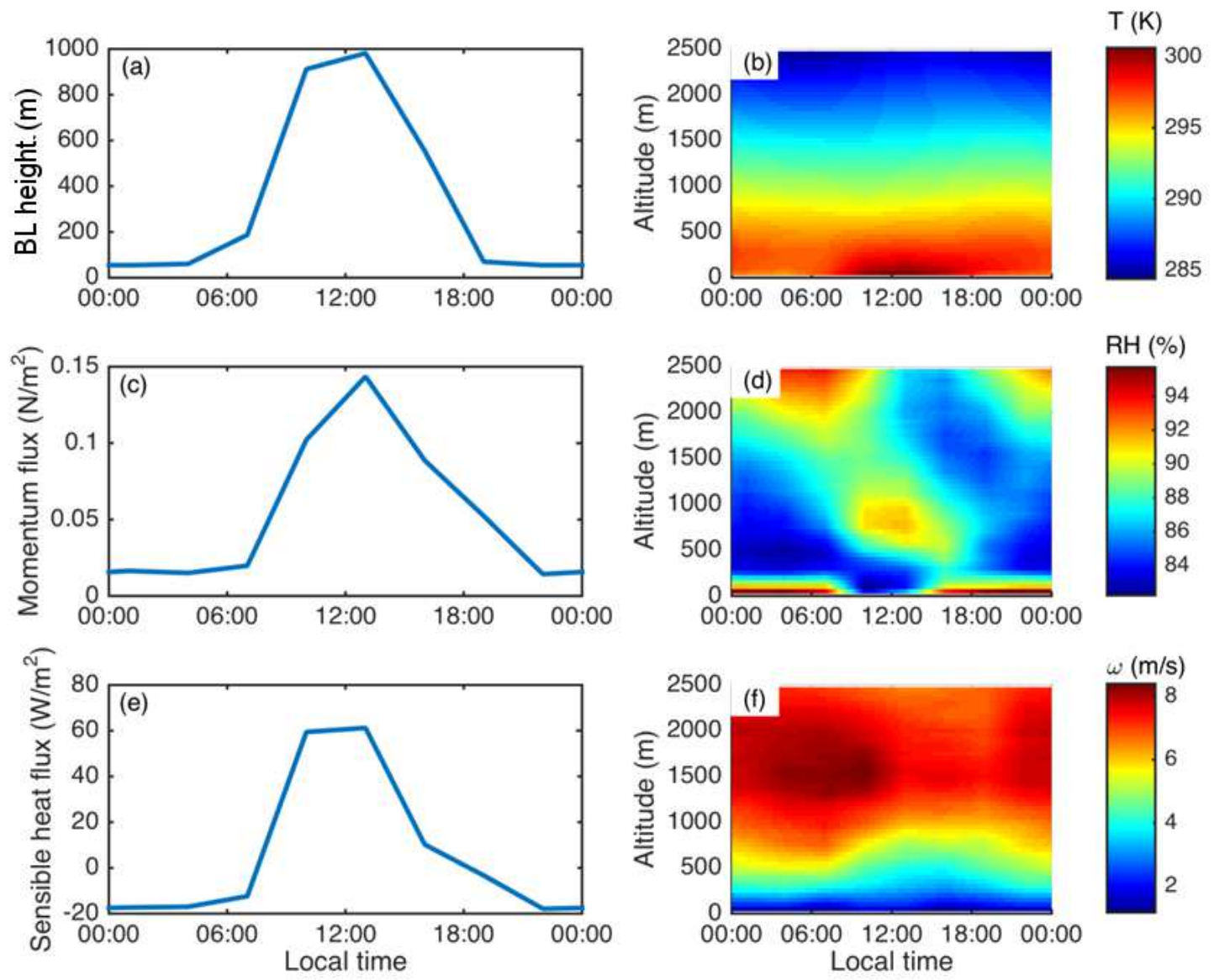

Figure S3. Median diurnal (a) boundary layer (BL) height, (b) temperature, (c) momentum flux, (d) relative humidity (RH), (e) sensible heat flux, and (f) horizontal wind speed $(\omega)$ during the wet season. The meteorological data obtained from Global Data Assimilation System (GDAS) represents the median conditions during the wet seasons of 2008 and 2009. 

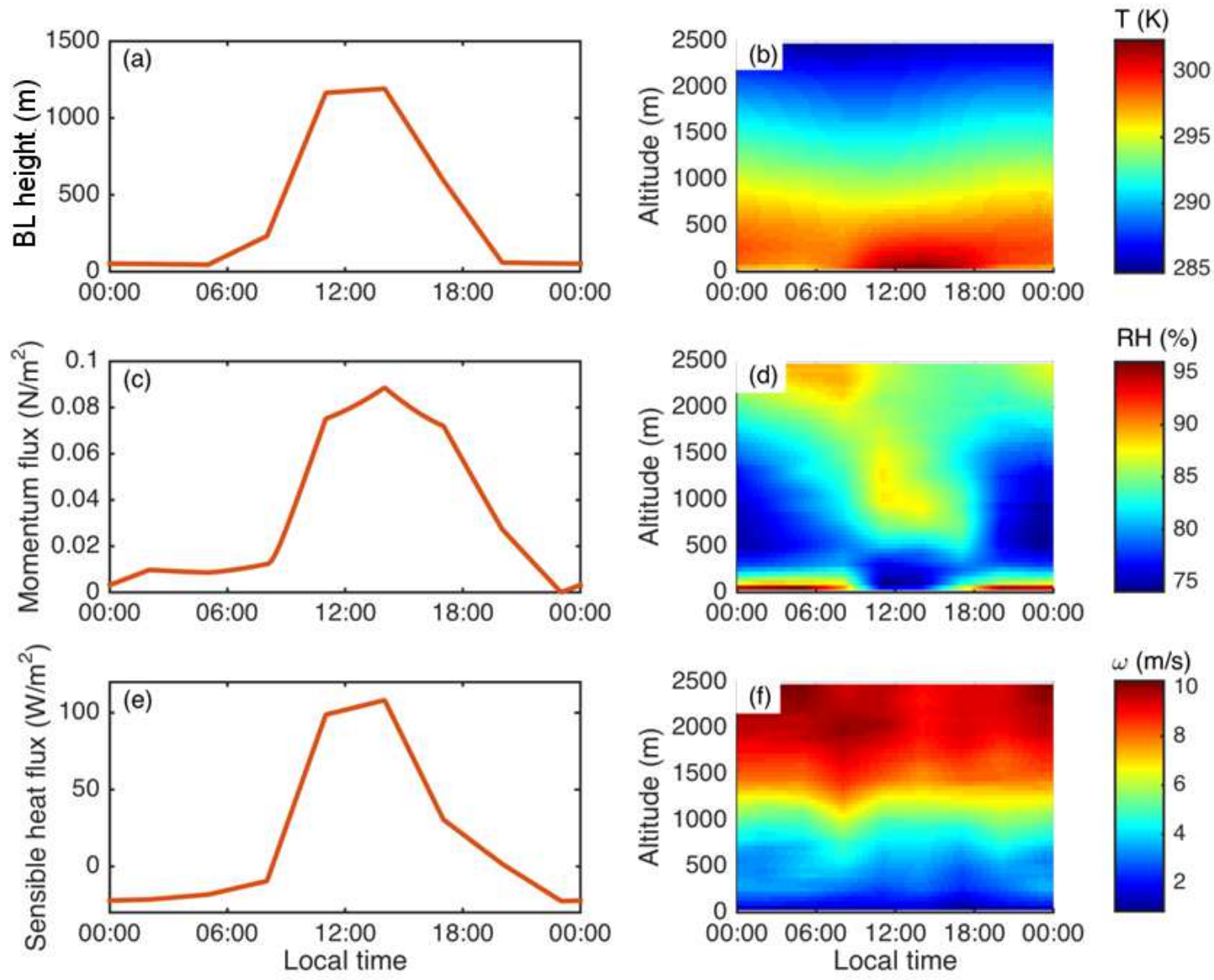

Figure S4. Median diurnal (a) boundary layer (BL) height, (b) temperature, (c) momentum flux, (d) relative humidity (RH), (e) sensible heat flux, and (f) horizontal wind speed $(\omega)$ during the wet season. The meteorological data obtained from Global Data Assimilation System (GDAS) represents the median conditions during the dry seasons of 2008 and 2009. 

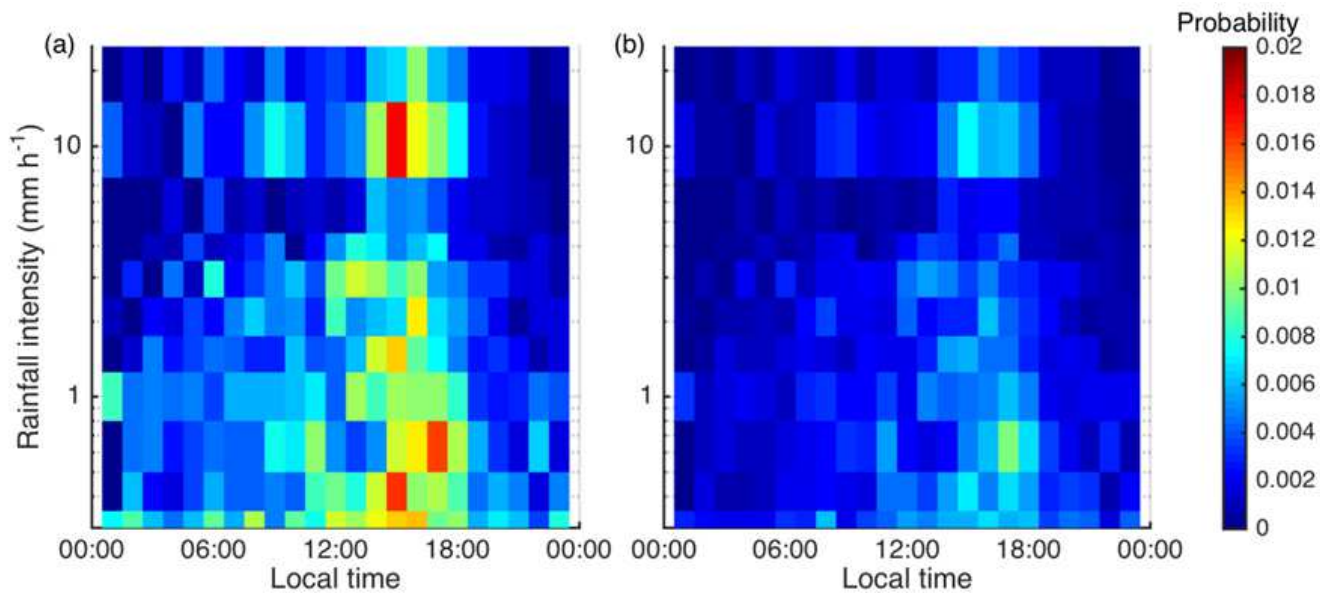

Figure S5. Probability of rainfall intensity at different times during the day for: (a) wet season and (b) dry season. Measurements were taken at the K34 tower site, $1 \mathrm{~km}$ away from the TT34 site, between 2008 and 2009. Data was provided by INPA (Brazilian National Institute for Amazonian Research). 


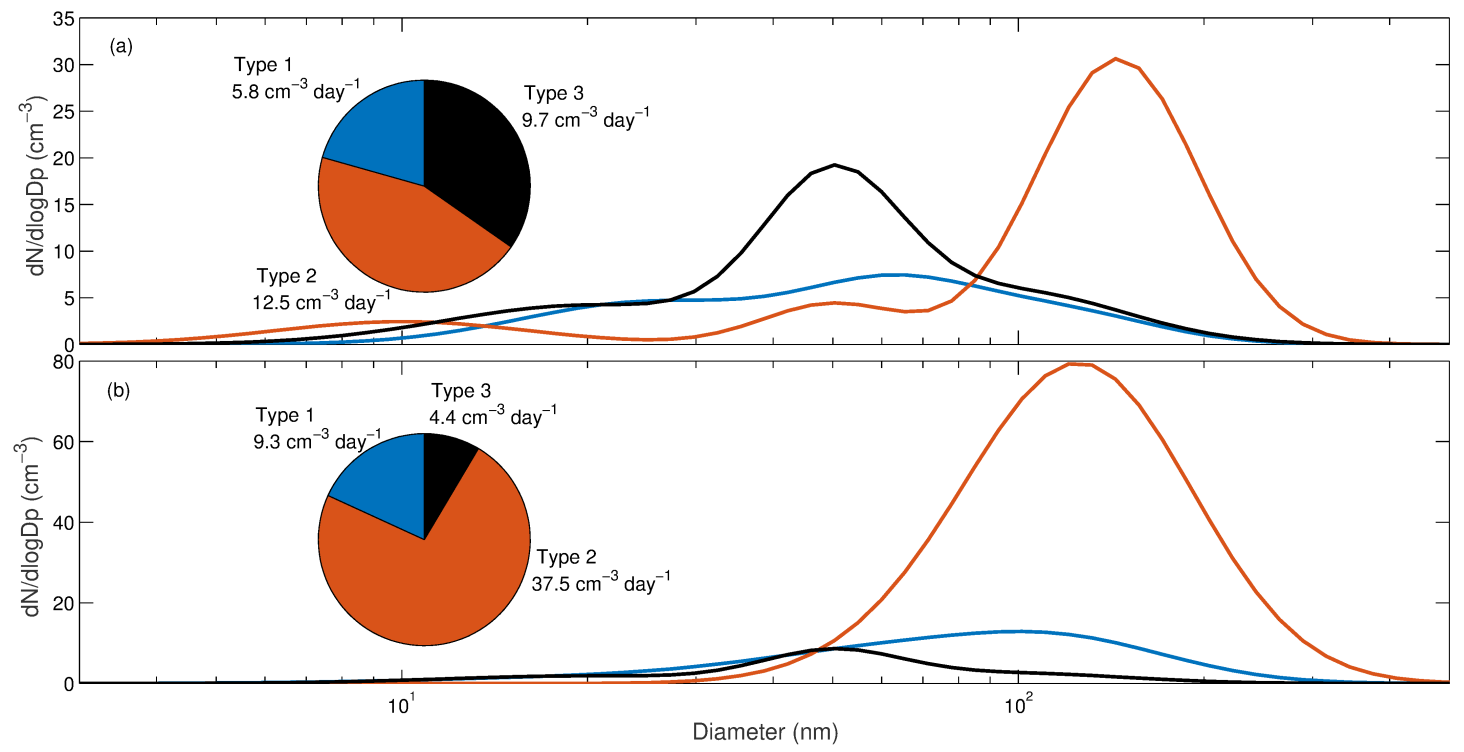

Figure S6. Modeled daily effective particle number size distribution emission contribution of the different particle source types to the lowermost $1000 \mathrm{~m}$ of the atmosphere. Type 1 refers to a local source of particles emitted at the surface; Type 2 to a source of particles that entrain into the BL during the morning; and Type 3 to a source of particles from convective downdrafts. The pie charts show the absolute total particle number concentration contributions of each particle source type. Panel (a) represents the wet season and panel (b) the dry season. 


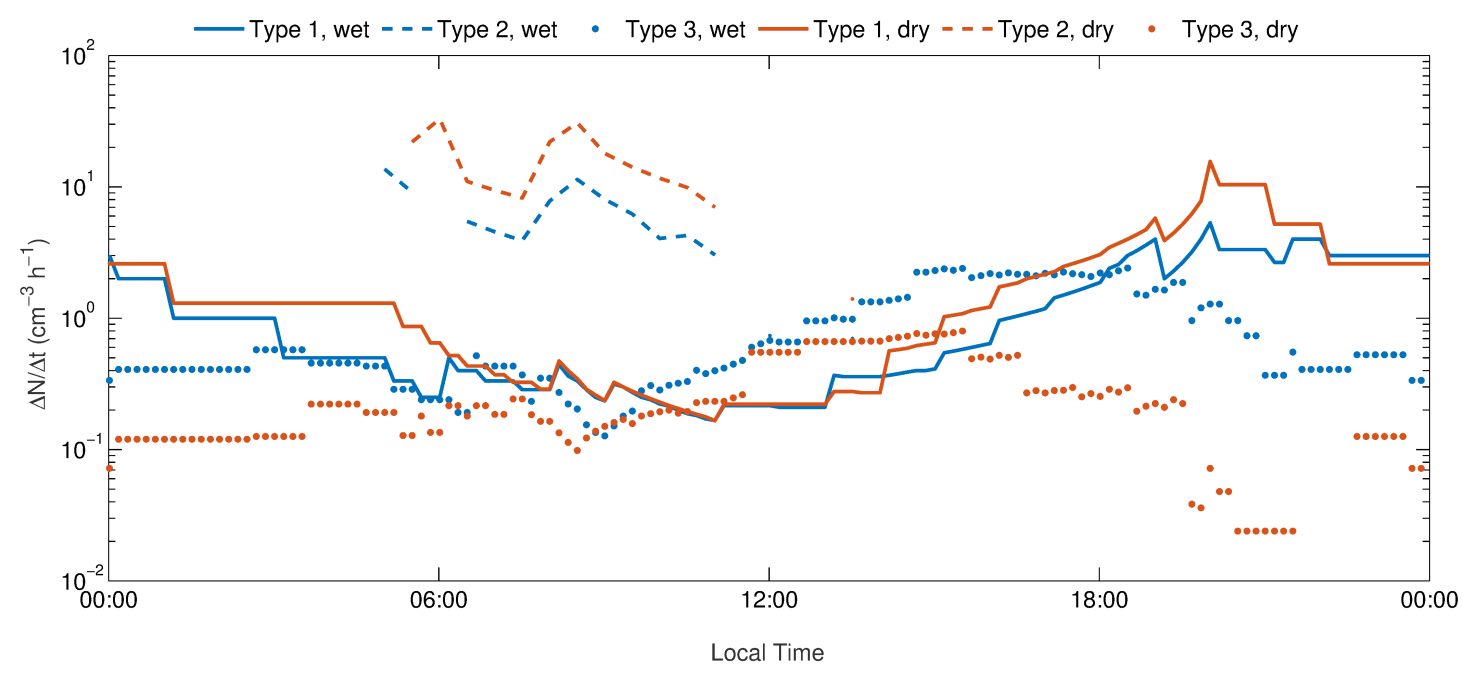

Figure S7. Modeled contribution of the different particle sources to the particle number concentrations in the BL at different times of the day, during the wet and dry season. Particle source Type 1 represents particles emitted at the surface from local sources; Type 2 refer to accumulation mode particles entrained into the BL in the morning; and Type 3 refer to particles that are mixed into the BL during convective downdrafts. 

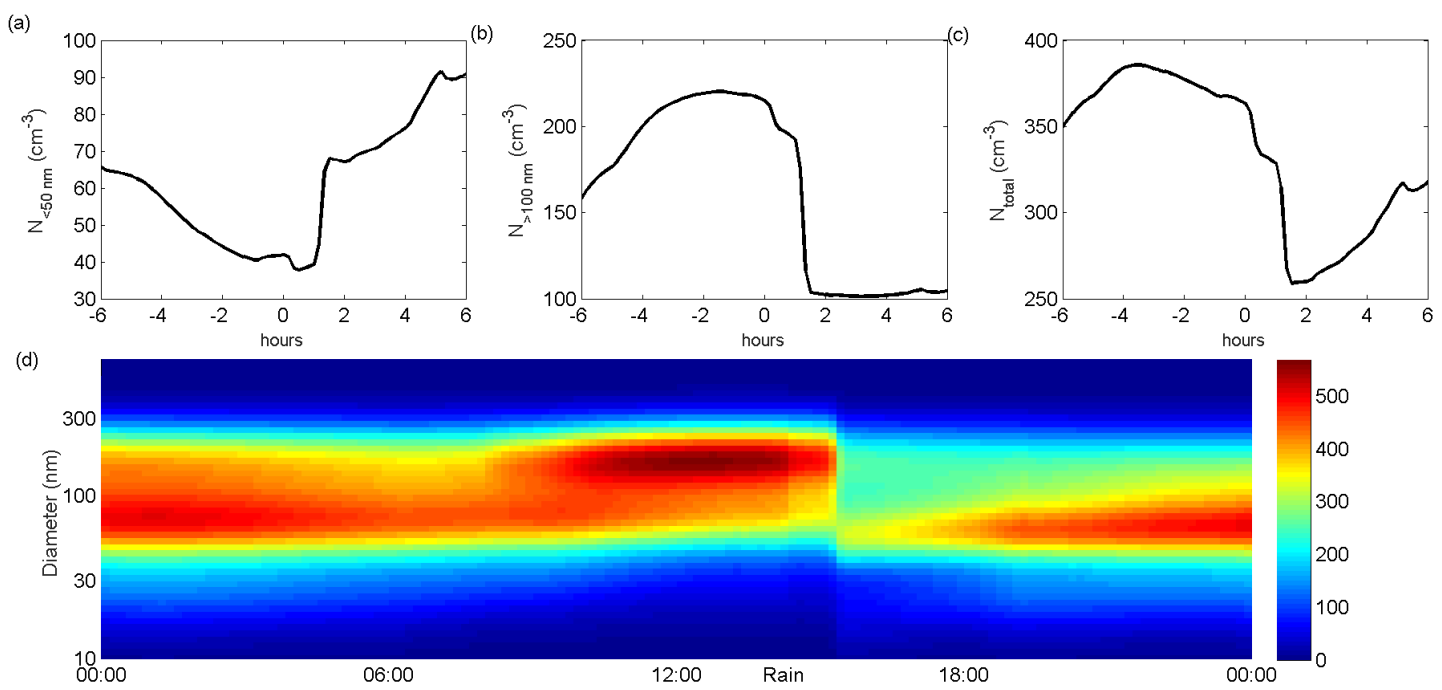

Figure S8. Model particle number size distribution evolution during a hypothetical convective precipitation event with a rainfall intensity of $20 \mathrm{~mm} \mathrm{~h}^{-1}$. The rainfall starts at 14:00 and ends at 14:15. The consecutive convective downdraft of FT aerosol particles into the BL is assumed to occur with a time lag of 1 hour after the rainfall start (15:00-15:15). Panel (a) shows the concentration change of particles smaller than $50 \mathrm{~nm}$ in diameter, (b) the concentration change of particles larger than $100 \mathrm{~nm}$ in diameter and (c) the total particle number concentration change. Panel (d) shows the particle number size distribution evolution during the complete day. Until 14:00 the modeled particle number size distribution resembles the median wet season diurnal variation. The increasing accumulation mode particle concentrations during the morning 08:00-12:00 is due to entrainment of accumulation mode particles during the buildup of the CBL. 

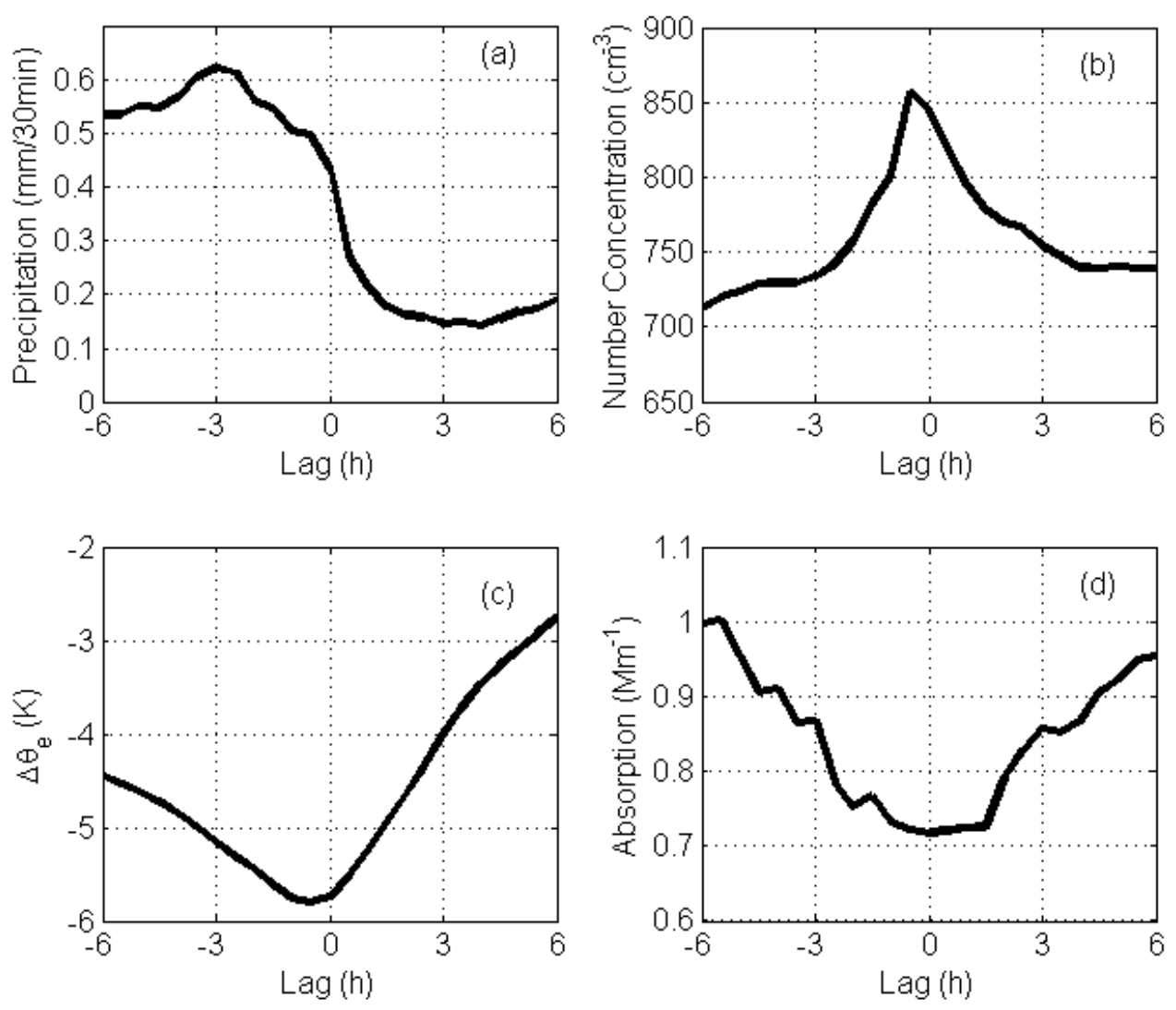

Figure S9. Lag time analysis referring to the time of occurrence of particle size distribution cluster \#1 (zero lag). The panels show mean values before and after the occurrence of cluster \#1 for (a) precipitation intensity, (b) total particle number concentration, (c) variations on equivalent potential temperature $\left(\Delta \theta_{e}\right)$ and (d) particle light absorption coefficients at $637 \mathrm{~nm}$. Negative and positive lags respectively denote hours before and after the occurrence of cluster \#1. Cluster \#1 occurred after rainy periods and at periods of negative $\Delta \theta_{e}$, indicating the occurrence of downdrafts. Cluster \#1 also occurred at periods of particle number concentrations fast increase and absorption coefficients decrease. 


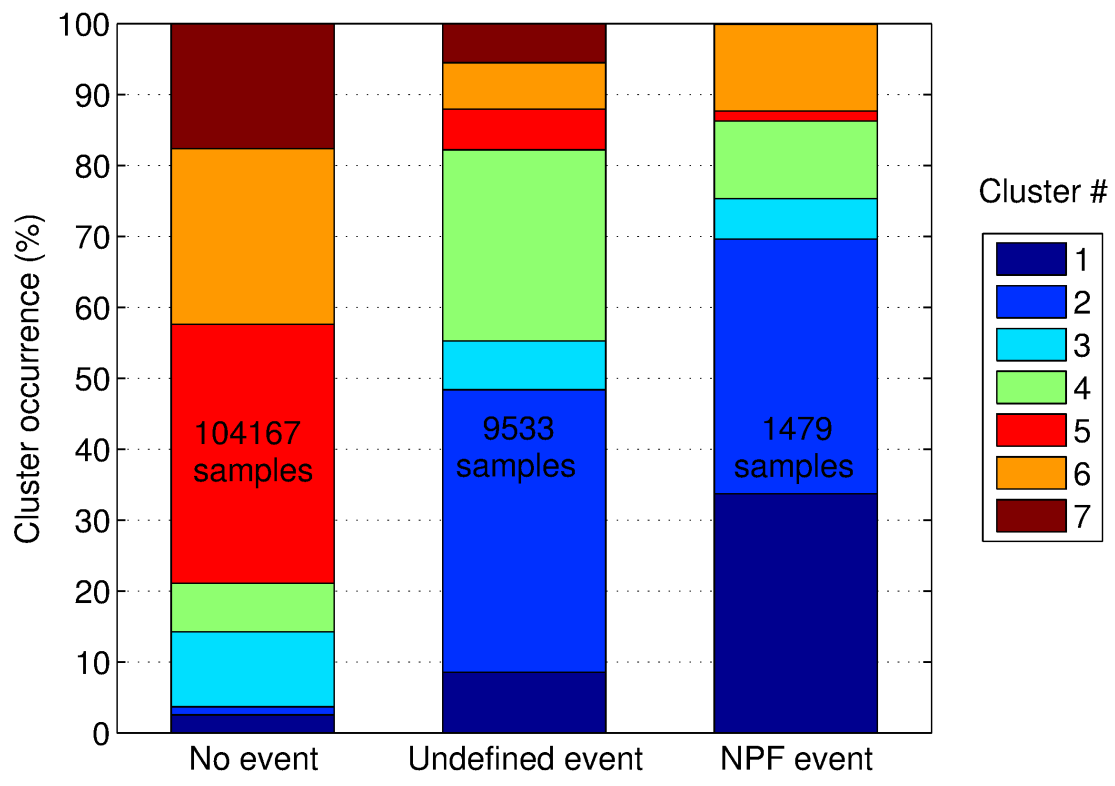

Figure S10. Percent occurrence of clusters in days classified as "no event" day, and during periods classified as "undefined event" and "new particle formation and growth" (NPF) event. 


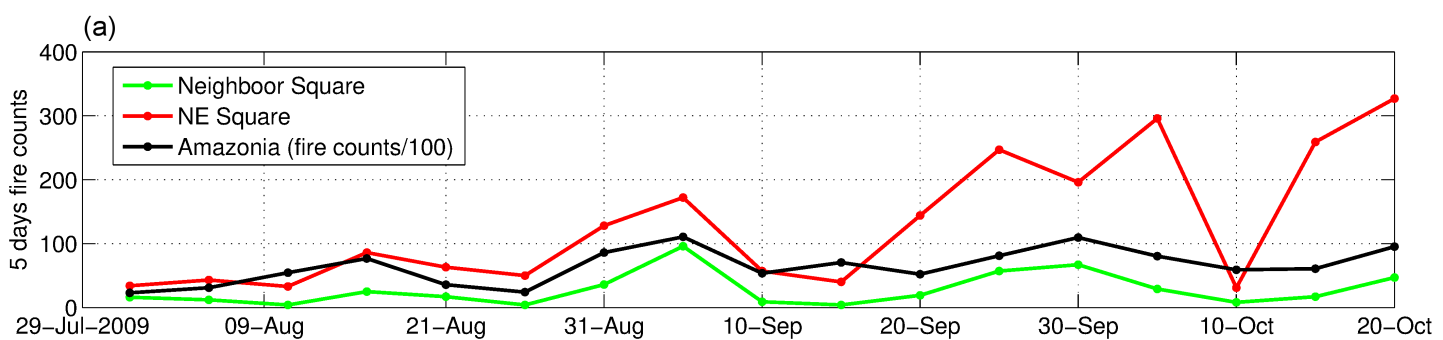

(b)
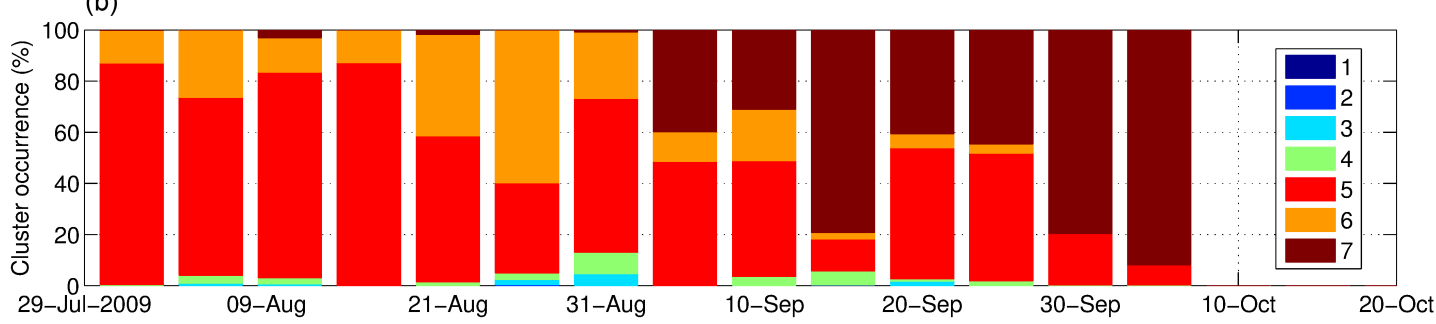

(c) $16-21$ Aug

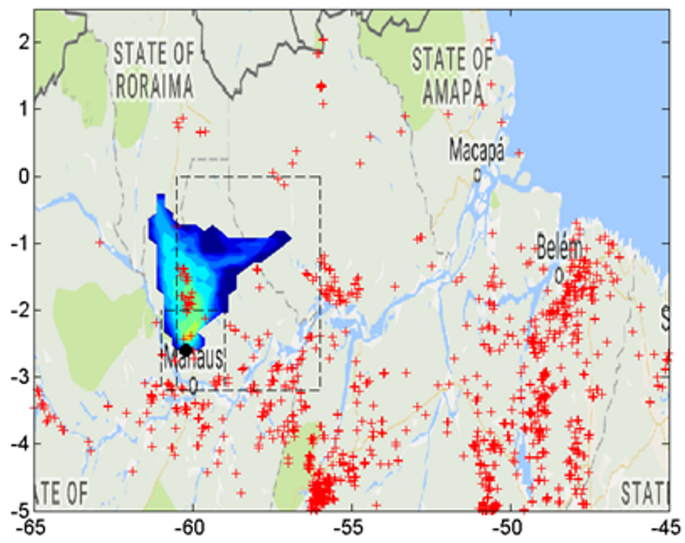

(d) $30 \mathrm{Sep}-050 \mathrm{ct}$

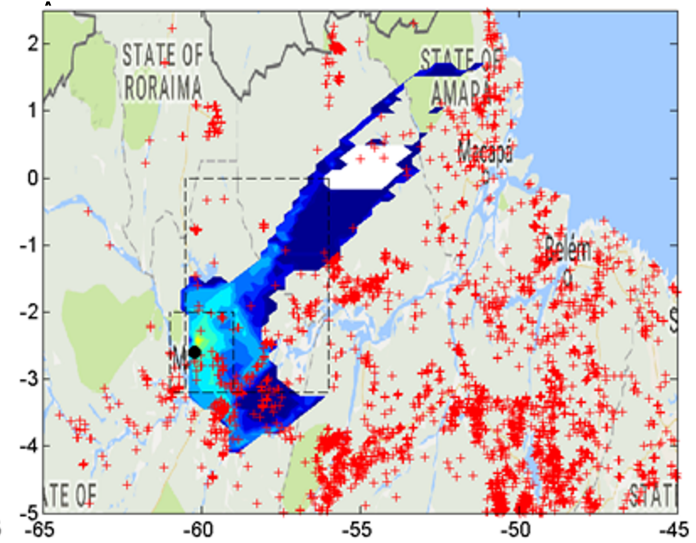

Figure S11. (a) Number of fire spots summed over five days in the Amazon Basin, in a square NE to the TT34 site, and in a square in the neighborhood of the TT34 site, observed between 01 Aug 2009 and 01 Jan 2010. (b) Five day percent occurrence of particle size distribution clusters. (c) Contourplot showing the density of Hysplit 48 hour back trajectories calculated for the period between 16-21 Aug 2009, when size distribution cluster \#5 was predominant. Warm colors represent frequent back trajectories paths ending at the TT34 site, symbolized by a black circle. Red crosses locates fire spots. Dashed lines delimit the NE square and the neighborhood square around the TT34 site. (d) Same for the period between 30 Sep and 05 Oct 2009, when size distribution cluster \#7 was predominant. 


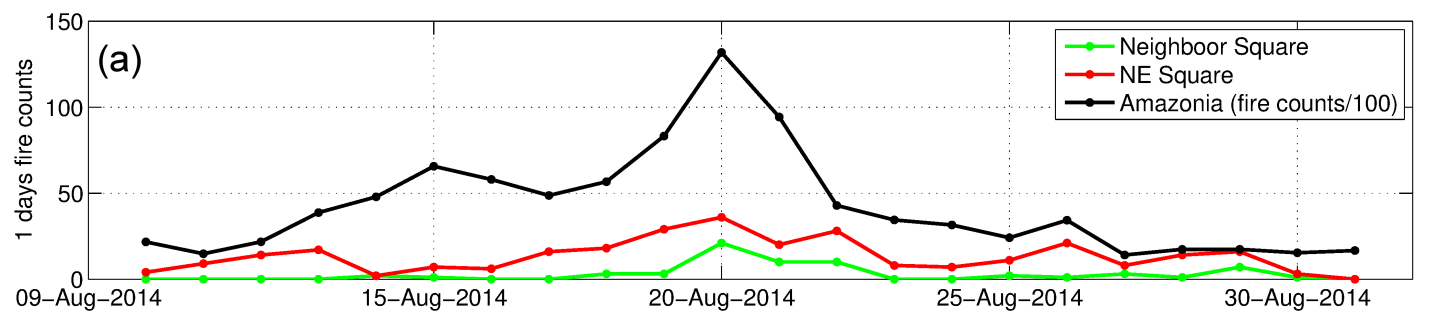

(b)

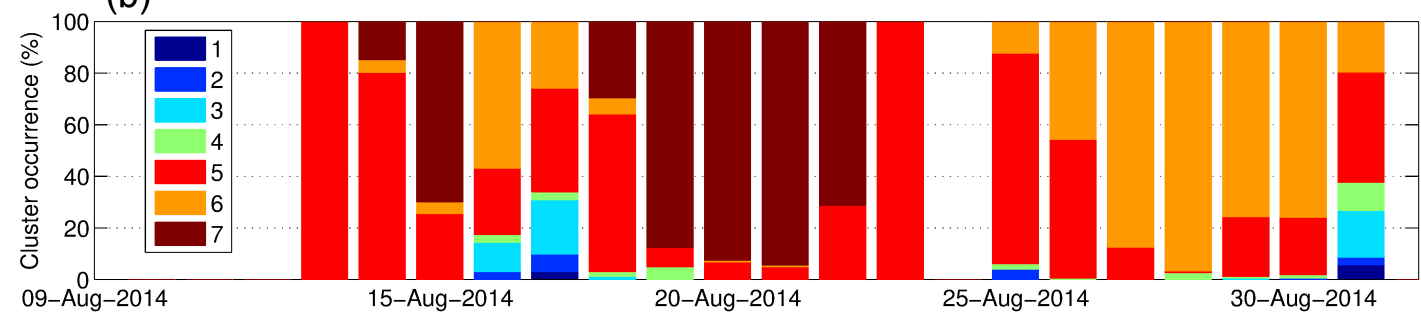

(c) $18-23$ Aug

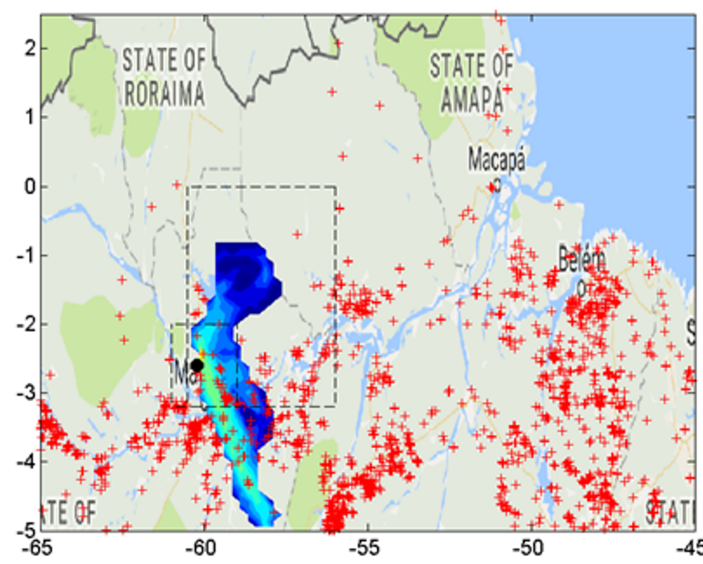

(d) 26-31 Aug

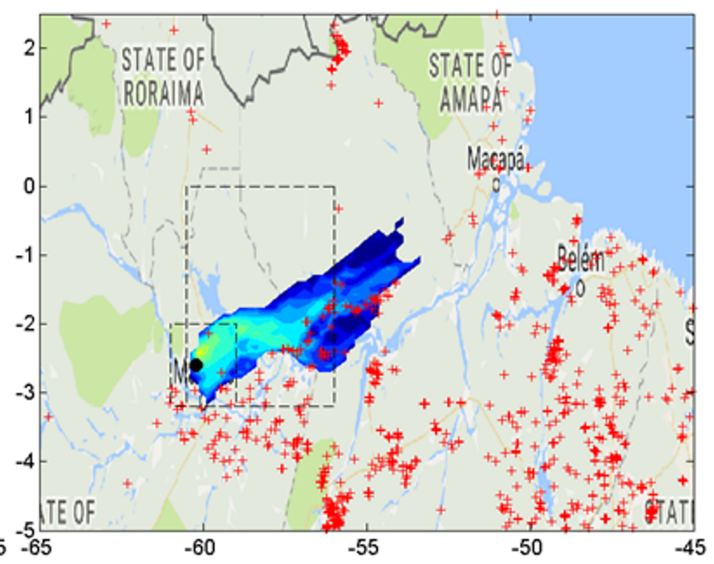

Figure S12. (a) Daily number of fire spots in the Amazon Basin, in a square NE to the TT34 site, and in a square in the neighborhood of the TT34 site, observed between 10 Aug and 01 Sep 2014. (b) Daily percent occurrence of particle size distribution clusters. (c) Contourplot showing the density of Hysplit 48 hour back trajectories calculated for the period between 18-23 Aug 2014, when size distribution cluster \#7 was predominant. Warm colors represent frequent back trajectories paths ending at the TT34 site, symbolized by a black circle. Red crosses locates fire spots. Dashed lines delimit the NE square and the neighborhood square around the TT34 site. (d) Same for the period between 26-31 Aug 2014, when size distribution clusters \#5 and \#6 were predominant. 

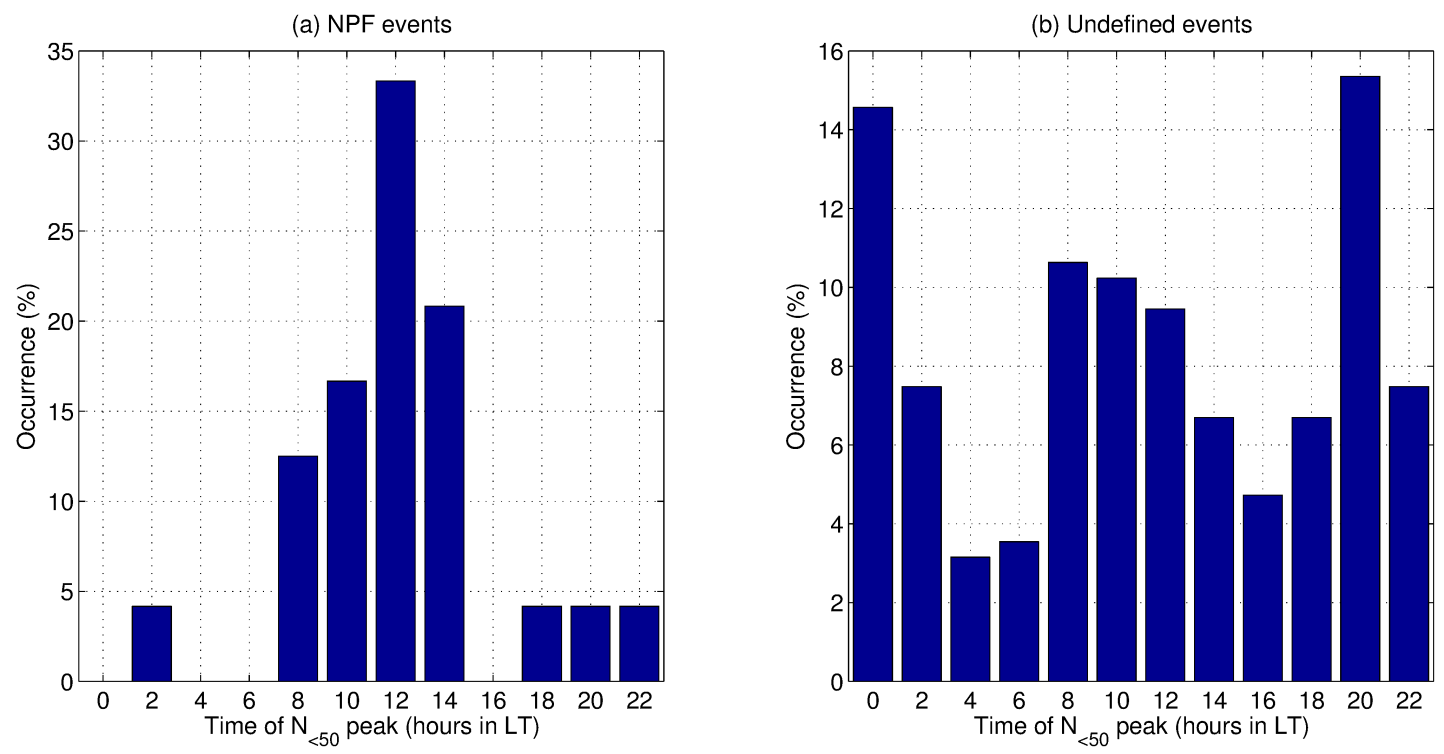

Figure S13. Histograms for the start time of NPF events (a) and undefined events (b). Start time was based on the instant at which peak concentrations of particles with diameter less than $50 \mathrm{~nm}\left(N_{<50}\right)$ were observed. 

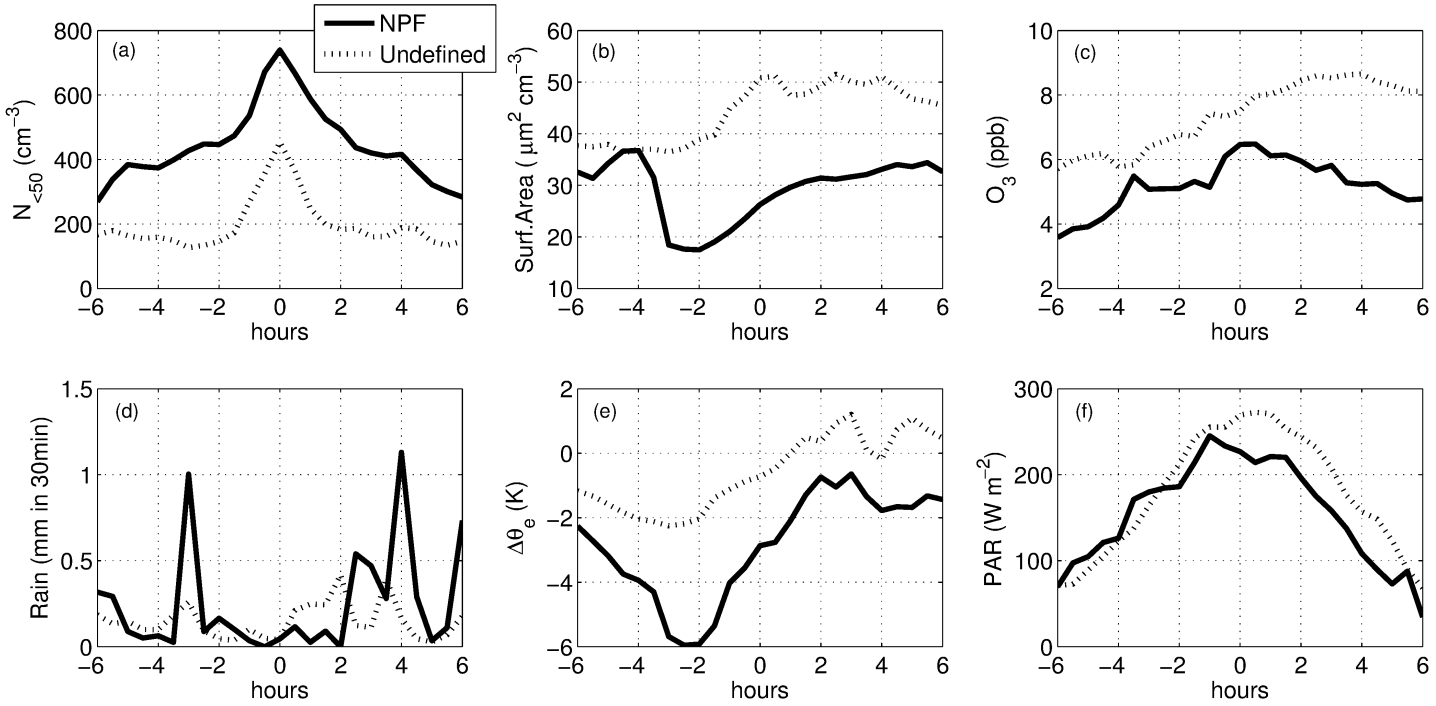

Figure S14. Mean values within \pm 6 hours from the start of new particle formation and growth (NPF) and undefined events: (a) concentration of particles with diameter less than $50 \mathrm{~nm}\left(N_{<50}\right)$; (b) particle surface area; (c) tropospheric ozone mixing ratios; (d) precipitation in 30 min; (e) variation on equivalent potential temperature $\left(\Delta \theta_{e}\right)$; (f) photosynthetic active radiation (PAR). Only diurnal events (starting between 8:00 and 16:00 local time) were considered in this plot. 

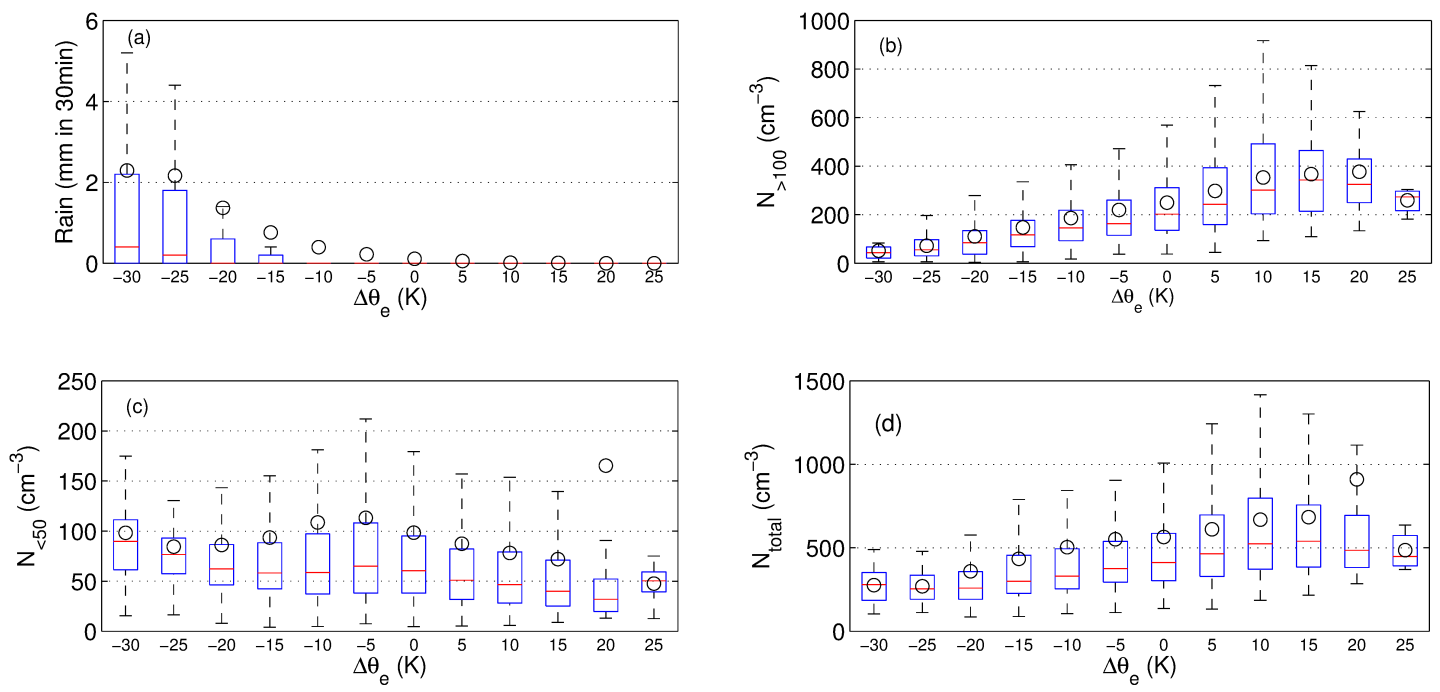

Figure S15. Boxplots showing different variables as a function of variations on equivalent potential temperature $\left(\Delta \theta_{e}\right)$, based on the method described by Wang et al. (2016): (a) precipitation in $30 \mathrm{~min}$; (b) number concentration of particles with diameter greater than $100 \mathrm{~nm}$ $\left(N_{>100}\right)$; (c) number concentration of particles with diameter smaller than $50 \mathrm{~nm}\left(N_{<50}\right)$; (d) total particle number concentration $\left(N_{\text {total }}\right)$. Red lines denote median values, black circles represents mean values, blue boxes represent quartiles and whiskers denote percentiles 10 and 90. Only wet season afternoon periods (12:00-16:00 local time) were considered in this plot. 